EPJ Web of Conferences 38, 12003 (2012)

DOI: $10.1051 /$ epjconf/20123812003

(C) Owned by the authors, published by EDP Sciences, 2012

\title{
Total and partial photoneutron reactions cross sections - new analysis and evaluation
}

\author{
B.S. Ishkhanov ${ }^{1,2}$, V.N. Orlin ${ }^{2}$, and V.V. Varlamov ${ }^{2}$ \\ ${ }^{1}$ Lomonosov Moscow State University, Physics Faculty, 199991 Moscow, Russia \\ ${ }^{2}$ Lomonosov Moscow State University, Skobeltsyn Institute of Nuclear Physics, 199234 Moscow, Russia
}

\begin{abstract}
The well-known problems of significant disagreements between partial photoneutron reactions $(\gamma, \mathrm{n}),(\gamma, 2 \mathrm{n})$ and $(\gamma, 3 \mathrm{n})$ cross sections data obtained in various experiments are discussed. Using new objective criteria of data reliability it was shown that majority of data under discussion contains significant distortions because of definite shortcomings of experimental photoneutron multiplicity sorting methods used - the majority of experimental data obtained such methods are not reliable. These criteria were transitional photoneutron multiplicity functions $F_{\mathrm{i}}=\sigma(\gamma, \mathrm{in}) / \sigma(\gamma, x \mathrm{n})$ having values not higher than $1.00,0.50,0.33,0.25$, $0.20 \ldots$, correspondingly for $\mathrm{i}=1,2,3,4,5, \ldots$ New experimentally-theoretical method was proposed to evaluate reliable data free from shortcomings of neutron multiplicity sorting. For partial reaction cross section evaluation the only experimental data for total neutron yield reaction cross section $\sigma^{\exp }(\gamma, x \mathrm{n})=\sigma^{\exp }[(\gamma, \mathrm{n})+$ $2(\gamma, 2 \mathrm{n})+3(\gamma, 3 \mathrm{n})+\ldots]$ free from the mentioned shortcomings are used together with functions $F_{\mathrm{i}}^{\text {theor }}$ calculated within the modern model for photonuclear reactions $-\sigma^{\text {eval }}(\gamma$, in $)=F_{\mathrm{i}}^{\text {theor }} \cdot \sigma^{\text {exp }}(\gamma, \mathrm{xn})$. Many new reliable data for ${ }^{90} \mathrm{Zr},{ }^{115} \mathrm{In},{ }^{112,114,116,117,118,119,120,122,124} \mathrm{Sn},{ }^{159} \mathrm{~Tb},{ }^{181} \mathrm{Ta},{ }^{197} \mathrm{Au}$, and ${ }^{208} \mathrm{~Pb}$ were evaluated. Deviations of evaluated data from experimental ones are analysed and the physical consequences are discussed.
\end{abstract}

\section{Introduction}

The problem of significant discrepancies between partial photoneutron reactions cross sections obtained in different experiments is as old and well-known as modern and very important.

Data on reactions with formation of various numbers of neutrons, primarily $(\gamma, n),(\gamma, 2 n)$, and $(\gamma, 3 n)$, were obtained as a rule many years ago and were included into various reviews [1], Atlases [2, 3] and databases [4]. They are using in many basic and applied research such as traditional investigations (Giant Dipole Resonance (GDR) main features, GDR configurational and isospin splitting, competition between statistical and direct processes in GDR decay channels, sum rule exhaustion etc.) as in actual new research (for example, for monitoring of the beam luminosity in ultra-relativistic heavy-ion colliders $[5,6]$ by measuring neutron emission rates in mutual electromagnetic dissociation of colliding nuclei - neutron emission (primarily $(\gamma, \mathrm{n})$ and $(\gamma, 2 \mathrm{n})$ rates in nuclei mutual electromagnetic dissociation).

The majority of such kind data has been obtained using quasi-monoenergetic $\gamma$-quanta produced by annihilation in flight of relativistic positronsat two laboratories - the National Lawrence Livermore Laboratory (USA) and the France Centre d'Etudes
Nucleaires de Saclay $[1,2]$. In both the laboratories the neutron multiplicity was obtained using their kinetic energy measurements but experimental methods were different. Those arrangement differences mean that cross section obtaining conditions are different and as result there are many complicated, curious, dramatic systematic discrepancies between partial photoneutron reaction cross sections obtained at Livermore and Saclay.

Briefly, without details - all $(\gamma, \mathrm{n})$ reaction cross sections are larger at Saclay but all $(\gamma, 2 n)$ ones quite the contrary at Livermore (Table 1). Those disagreements were the subject of special investigations during many years.

\section{Short history of problem solving}

Because data of both laboratories agree as to total number of neutrons detected, the differences in their reaction cross sections $\sigma(\gamma, \mathrm{n})$ and $\sigma(\gamma, 2 \mathrm{n})$ were proposed [7] to arise from the separation of counts into $1 \mathrm{n}$ and $2 \mathrm{n}$ events (neutron multiplicity sorting). In [7] the results obtained using neutron multiplicity sorting were compared with those obtained using alternative method of induced activity in which concrete partial reactions are identified using detection of not outgoing neutrons but of formatting final nucleus. That was shown that results of 
measurements for ${ }^{181} \mathrm{Ta}$ of $\sigma(\mathrm{e}, \mathrm{xn})$ and $\sigma(\mathrm{e}, \mathrm{n})$ reaction cross sections (measured directly by activation method for $98.3 \mathrm{keV} \gamma$-ray line from decay of final nucleus ${ }^{180} \mathrm{Ta}$ $\rightarrow{ }^{180} \mathrm{Hf}$ ) recalculated using virtual photon spectra into correspondent $\sigma(\gamma, \mathrm{xn})$ and $\sigma(\gamma, \mathrm{n})$ reaction cross sections lead to agreement with Livermore data but not with Saclay data: Saclay $\sigma(\gamma, 2 \mathrm{n})$ data are significantly underestimated and therefore $\sigma(\gamma, n)$ data correspondingly overestimated because some events from $(\gamma, 2 \mathrm{n})$ reaction at Saclay were interpreted as two $(\gamma, n)$ events.

Table 1. Some examples [8] of Saclay/Livermore integrated cross sections ratios for $(\gamma, n)$ and $(\gamma, 2 n)$ reactions.

\begin{tabular}{|c|c|c|}
\hline Nucleus & $\begin{array}{c}\sigma^{\text {jnt }}{ }_{\mathbf{S}}(\boldsymbol{\gamma}, \mathbf{n}) / \sigma^{\text {jnt }}{ }_{\mathbf{L}}(\boldsymbol{\gamma}, \mathbf{n}), \\
\text { arb.units }\end{array}$ & $\begin{array}{c}\sigma_{\mathbf{S}}^{\text {jnt }}(\boldsymbol{\gamma}, \mathbf{2 n}) / \sigma^{\text {jnt }}{ }_{\mathbf{L}}(\boldsymbol{\gamma}, \mathbf{2 n}), \\
\text { arb.units }\end{array}$ \\
\hline${ }^{51} \mathrm{~V}$ & 1.07 & 0.79 \\
\hline${ }^{89} \mathrm{Y}$ & 1.25 & 0.87 \\
\hline${ }^{90} \mathrm{Zr}$ & 1.26 & 0.73 \\
\hline${ }^{118} \mathrm{Sn}$ & 1.07 & 0.86 \\
\hline${ }^{127} \mathrm{I}$ & 1.34 & 1.07 \\
\hline${ }^{159} \mathrm{~Tb}$ & 1.07 & 0.71 \\
\hline${ }^{165} \mathrm{Ho}$ & 1.20 & 1.05 \\
\hline${ }^{181} \mathrm{Ta}$ & 1.25 & 0.89 \\
\hline${ }^{197} \mathrm{Au}$ & 1.00 & 0.69 \\
\hline
\end{tabular}

This point of view give to one possibility for simple, clear and direct way for correction the situation and evaluation of "correct" $\sigma(\gamma, 2 \mathrm{n})$ obtained at Saclay $\sigma^{2 \mathrm{n}}{ }_{\mathrm{s}}{ }^{*}$ which must put into agreement with "corrected" (multiplied by $R=\sigma^{\text {int }} \mathrm{s}(\gamma, \mathrm{xn}) / \sigma^{\text {int }}{ }_{\mathrm{L}}(\gamma, \mathrm{xn})$ ) data obtained at Livermore $R \sigma^{2 \mathrm{n}}{ }_{\mathrm{L}}$ - extra neutrons must be removed from $\sigma(\gamma, \mathrm{n})$ and put into $\sigma(\gamma, 2 \mathrm{n})$ :

$\sigma_{\mathrm{S}}^{2 \mathrm{n}}=\sigma_{\mathrm{S}}^{2 \mathrm{n}}+1 / 2\left(\sigma_{\mathrm{S}}^{\mathrm{n}}-R \sigma_{\mathrm{L}}^{\mathrm{n}}\right)=R \sigma_{\mathrm{L}}^{2 \mathrm{n}}$.

Such approach was used in [7] for evaluation of corrected data for 12 nuclei $\left({ }^{89} \mathrm{Y},{ }^{115} \mathrm{In},{ }^{117,118,120,124} \mathrm{Sn}\right.$, $\left.{ }^{133} \mathrm{Cs},{ }^{159} \mathrm{~Tb},{ }^{165} \mathrm{Ho},{ }^{181} \mathrm{Ta},{ }^{197} \mathrm{Au},{ }^{208} \mathrm{~Pb}\right)$ and later in [8] for their reevaluation and new evaluations for additional 7 nuclei $\left({ }^{51} \mathrm{~V},{ }^{75} \mathrm{As},{ }^{90} \mathrm{Zr}{ }^{116} \mathrm{Sn},{ }^{127} \mathrm{I},{ }^{232} \mathrm{Th},{ }^{238} \mathrm{U}\right)$. Conclusions of such investigations carried out have been formulated as following:

-Photoneutron multiplicity sorting was correct at Livermore but not at Saclay;

- $\sigma(\gamma, 2 \mathrm{n})$ obtained at Saclay are significantly underestimated and, correspondingly, $\sigma(\gamma, \mathrm{n})$ - overestimated;

- "correct" Livermore data could be used after simple renormalization $\left(R \sigma^{2 n}\right)$ but for "incorrect" Saclay data the serious recalculation (1) should be used.

So the old well-known problem of systematic disagreements under discussion seemed to be solved. But after more detailed investigations the new problem appeared.

\section{Objective criteria for data reliability and authenticity}

Because very strange behavior of some $\sigma(\gamma, \mathrm{n})$ obtained at Livermore in their vanishing above $(\gamma, 2 \mathrm{n})$ reaction threshold $B 2 \mathrm{n}$ the new problem of reliability and authenticity of "correct" Livermore data appeared.

One typical example of ${ }^{116} \mathrm{Sn}(\gamma, \mathrm{n}){ }^{115} \mathrm{Sn}$ cross section obtained at Livermore [9] behavior is presented in figure 1a.

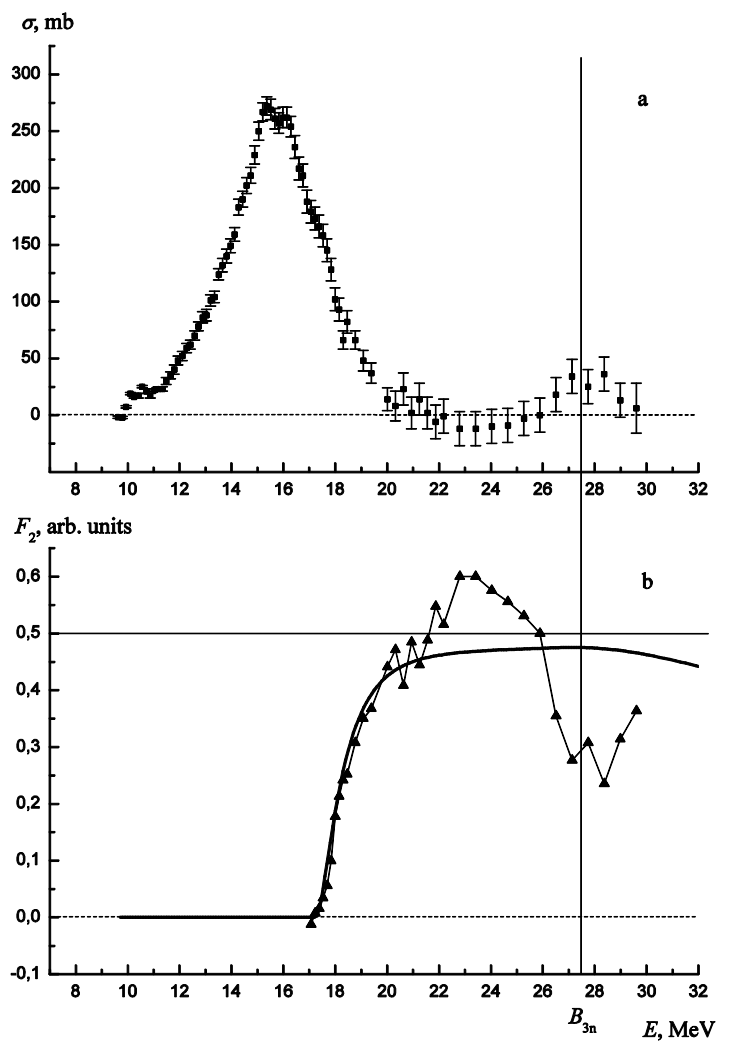

Fig. 1. Typical example of non-physical behaviour of cross section of the reaction ${ }^{116} \mathrm{Sn}(\gamma, \mathrm{n}){ }^{115} \mathrm{Sn}$ [9] (a) and of correspondent transitional multiplicity function (b) $-F_{2}{ }^{\exp }[10]$, triangles and $F_{2}^{\text {theor }}[11,12]$, line.

One can see that above the GDR maximum $\sigma(\gamma, n)$ falls rapidly going into the region of negative values, than goes again to positive values showing clear positive maximum. This is not natural for typical resonance behavior of photonuclear reaction cross section.

In figure $1 \mathrm{~b}$ there are presented experimental and theoretical data for the specially proposed [10] transition multiplicity function $F_{2}$ which is an objective very simple, clear, direct and absolute criterion of data reliability and authenticity of three reaction cross sections - $\sigma(\gamma, \mathrm{n}), \sigma(\gamma, 2 \mathrm{n})$ and $\sigma(\gamma, 3 \mathrm{n})-$ at the same time

$$
\begin{gathered}
F_{2}=\sigma(\gamma, 2 \mathrm{n}) / \sigma(\gamma, \mathrm{xn})= \\
=\sigma(\gamma, 2 \mathrm{n}) / \sigma[(\gamma, \mathrm{n})+2(\gamma, 2 \mathrm{n})+3(\gamma, 3 \mathrm{n})+\ldots] .
\end{gathered}
$$


The point is that following the definition (2) $F_{2}=$ const $=0.50$ without $\sigma(\gamma, \mathrm{n})$ and $\sigma(\gamma, 3 \mathrm{n})$ contributions into $\sigma(\gamma, \mathrm{xn})$. Deviation from the line "const $=0.50$ " at low energies is due to the high-energy tail of $\sigma(\gamma, \mathrm{n})$, at high energies - due to presence of the front of $\sigma(\gamma, 3 \mathrm{n})$. From Eq. (2) it is absolutely clear that " $F_{2}>0.50$ " means that multiplicity sorting has been done incorrectly.

Figure $1 \mathrm{~b}$ shows that the cross section of the reaction ${ }^{116} \mathrm{Sn}(\gamma, 2 \mathrm{n}){ }^{114} \mathrm{Sn}[9]$ in the energy region $E=21.5-26.0$ $\mathrm{MeV}$ (where " $\left.F_{2}{ }^{\exp }>0.50 "\right)$ strongly correlates with the strange $\sigma(\gamma, \mathrm{n})$ negative, i.e. non-physical, values.

Moreover, results of theoretical calculations of $F_{2}{ }^{\text {theor }}$ within the modern pre-equilibrium model of photonuclear reactions [11, 12] based on the Fermi-gas densities equations and taking into account the effects of nuclear deformation and GDR isospin splitting are presented in figure $1 \mathrm{~b}$.

One can see that behavior of $F_{2}{ }^{\text {theor }}$ difference from that of $F_{2}{ }^{\text {exp }}$ is pure physical:

- Below $B 2$ n only $(\gamma, \mathrm{n})$ is possible and $F_{2}=0$;

- Above $B 2 \mathrm{n}(\gamma, 2 \mathrm{n})$ reaction is open, $F_{2}{ }^{\text {theor }}$ increases (in accordance with competition between decreasing $\sigma(\gamma, \mathrm{n})$ and increasing $\sigma(\gamma, 2 \mathrm{n}))$, going to " 0.50 " top boundary, nowhere reaching it because of $\sigma(\gamma, \mathrm{n})$ tail presence;

- Above threshold $B 3 \mathrm{n}(\gamma, 3 \mathrm{n})$ reaction is open, $F_{2}{ }^{\text {theor }}$ decreases because of $3 \sigma(\gamma, 3 \mathrm{n})$ contribution appearance.

In systematic research carried out $[10,13-15]$ that have been found out that analogous non-physical reliable behavior of $\sigma(\gamma, \mathrm{n})$ and $F_{2}{ }^{\text {theor }}$ is typical for many data obtained at Livermore $\left({ }^{90,91,94} \mathrm{Zr},{ }^{115} \mathrm{In},{ }^{112,114,116,117-120} \mathrm{Sn}\right.$, $\left.{ }^{122,124} \mathrm{Sn},{ }^{127} \mathrm{I},{ }^{159} \mathrm{~Tb},{ }^{165} \mathrm{Ho},{ }^{181} \mathrm{Ta},{ }^{188,189} \mathrm{Os},{ }^{197} \mathrm{Au},{ }^{208} \mathrm{~Pb}\right)$. Several examples of main features of that research are presented in Table 2.

Table 2.Examples of $\sigma(\gamma, \mathrm{n})$ and $F_{2}{ }^{\text {theor }}$ non-physical behaviour.

\begin{tabular}{|c|c|c|}
\hline Nucleus [Ref] & $\begin{array}{c}\text { Negative } \sigma(\gamma, \mathrm{n}) \text { values } \\
\text { energy range, } \mathrm{MeV}\end{array}$ & $\begin{array}{c}\mathrm{F}_{2}{ }^{\text {exp}}-\max \\
\text { value }\end{array}$ \\
\hline${ }^{91} \mathrm{Zr}[16]$ & $23.0-30.0$ & 0.80 \\
\hline${ }^{94} \mathrm{Zr}[16]$ & $21.5-27.0$ & 0.70 \\
\hline${ }^{115} \mathrm{In}[9]$ & $20.5-31.0$ & 0.60 \\
\hline${ }^{116} \mathrm{Sn}[9]$ & $21.5-26.0$ & 0.62 \\
\hline${ }^{159} \mathrm{~Tb}[17]$ & $18.5-22.0$ & 0.60 \\
& $26.0-30.0$ & $2.00(!)$ \\
\hline${ }^{181} \mathrm{Ta}[18]$ & $17.0-22.5$ & 0.55 \\
& $22.5-24.0$ & 0.75 \\
\hline${ }^{180} \mathrm{Os}[19]$ & $18.0-21.0$ & 0.60 \\
\hline${ }^{208} \mathrm{~Pb}[20]$ & $17.5-26.5$ & 0.65 \\
\hline
\end{tabular}

Because such non-physical behavior of $\sigma(\gamma, n)$ and $F_{2}{ }^{\text {theor }}$ is typical for many nuclei investigated at Livermore there are many serious doubts that "correct" Livermore data for $\sigma(\gamma, \mathrm{n})$ and therefore for $\sigma(\gamma, 2 \mathrm{n})$ also are really correct. In addition to conclusions concern "incorrect" Saclay data have been made before $[7,8]$ that means that practically we have no reliable data.
This forced us to investigate data reliability and authenticity in details using objective criteria. It is evident that in addition to the criterion $F_{2}<0.50$ mentioned above the analogous criteria $F_{1}<1.00, F_{3}<$ $0.33, F_{4}<0.25, F_{5}<0.20$, etc. could be proposed [13].

Figure 2 presents $F_{1,2,3}$ criteria for experimental and theoretical data obtained for ${ }^{208} \mathrm{~Pb}$.
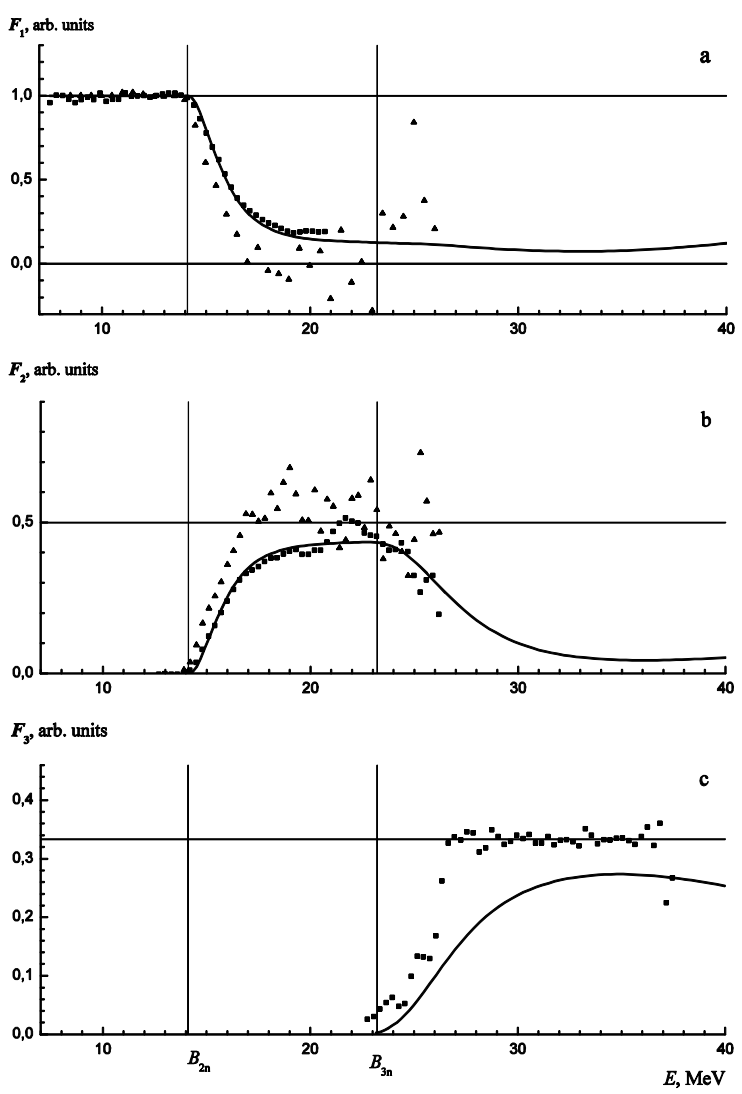

Fig. 2. Comparison of experimental Livermore ([20], triangles) and Saclay ([21], squares) $F_{1,2,3}$ exp data for ${ }^{208} \mathrm{~Pb}$ with correspondent theoretical results $F_{1,2,3}{ }^{\text {theor }}$ ([11, 12], line).

One can see that experimental neutron multiplicity sorting has been done incorrectly:

- In the energy range $\sim 17.5-23.0 \mathrm{MeV} F_{1}{ }^{\exp }$ has negative values;

- In the same energy range $\sim 17.5-23.0 \mathrm{MeV} F_{2}{ }^{\text {exp }}$ has values larger 0.50 ;

- At energies $\geq 26 \mathrm{MeV}$ values of $F_{3}{ }^{\text {exp }}$ are very close to 0.33 , that means that there are no the neutrons with multiplicity 1 and 2 (all such neutrons were erroneously attributed to $\sigma(\gamma, 3 \mathrm{n}))$.

It is very important to point out that for ${ }^{208} \mathrm{~Pb}$ in contradiction with mentioned above conclusions and recommendations [7,8], the results of our previous systematic research $[10,13-15]$, and absolutely unexpected Saclay data for both $\sigma(\gamma, \mathrm{n})$ and $\sigma(\gamma, 2 \mathrm{n})$ cross sections are reliably agree with theoretical results (although for $\sigma(\gamma, 3 n)$ the Saclay data disagree significantly). This strongly complicates the problem of data reliability - that means that all known data should be reanalyzed individually. 


\section{New approach to reliable data evaluation}

In systematic analyses [10,13-15] of well-known data on partial photoneutron reaction cross sections it was found out that there are many such kinds of data which reliability and authenticity are very doubtful. There are many energy regions where cross sections have nonphysical negative values and/or objective criteria $F_{2,3}$ exp have values larger than physically permitted limits -0.50 and 0.33 , respectively.

Therefore the use of such unreliable data could lead to doubtful conclusions in various applications. So the problem of development of the method for reliable data evaluation is very actual and important.

Since the main reason of disagreements and unreliability under discussion are connected with experimental methods of neutron multiplicity sorting the new so-called "experimentally-theoretical" approach for data evaluation free from such methods shortcomings has been proposed [10, 13-15].

The new approach uses as initial experimental data only that for total neutron yield reaction cross section $\sigma(\gamma, \mathrm{xn})=\sigma(\gamma, \mathrm{n})+2(\gamma, 2 \mathrm{n})+3(\gamma, 3 \mathrm{n})+\ldots]$ and for separation of that into partial reaction cross sections transition multiplicity functions $F_{1,2,3}$ theor calculated within the modern exciton pre-equilibrium photonuclear reaction model $[11,12]$ based on Fermi-gas densities and taking into account the effects of nucleus deformation and GDR isospin splitting. So the reliable reaction cross section is evaluated by the way

$\sigma^{\text {eval }}(\gamma$, in $)=F_{\mathrm{i}}^{\text {theor }}(\gamma$, in $) \cdot \sigma^{\exp }(\gamma, \mathrm{xn})$.

So competition of partial reactions is in accordance with equations of model and the sum of evaluated cross sections is equal to the experimental data for total neutron yield cross section.

Figure 3 illustrates the deviations of valuated cross sections from experimental ones for three partial reactions on ${ }^{159} \mathrm{~Tb}$.

One should pay attention to that that data evaluated for conditions free from experimental neutron multiplicity sorting methods shortcomings deviate from experimental cross sections significantly. Table 3 presents ratios of correspondent integrated cross sections for $\sigma(\gamma, \mathrm{n})$ and $\sigma(\gamma, 2 \mathrm{n})$ for ${ }^{159} \mathrm{~Tb}$.

One can see that evaluated $\sigma(\gamma, \mathrm{n})$ is $19 \%$ smaller than the Saclay experimental data but $18 \%$ larger than the Livermore ones. At the same time $\sigma(\gamma, 2 \mathrm{n})$ is $15 \%$ larger than the Saclay experimental data but $22 \%$ smaller than the Livermore once.

So the ratio $\sigma^{\text {int }}(\gamma, 2 \mathrm{n}) / \sigma^{\text {int }}(\gamma, \mathrm{n})$ which is very important for estimation of probabilities of many physical processes increased in $27 \%$ compare to the Saclay data and decreased in $30 \%$ compare to the Livermore ones. That forces one to re-evaluate many physical effects, i.e. a competition between statistical and direct process probabilities in various GDR decay channels.

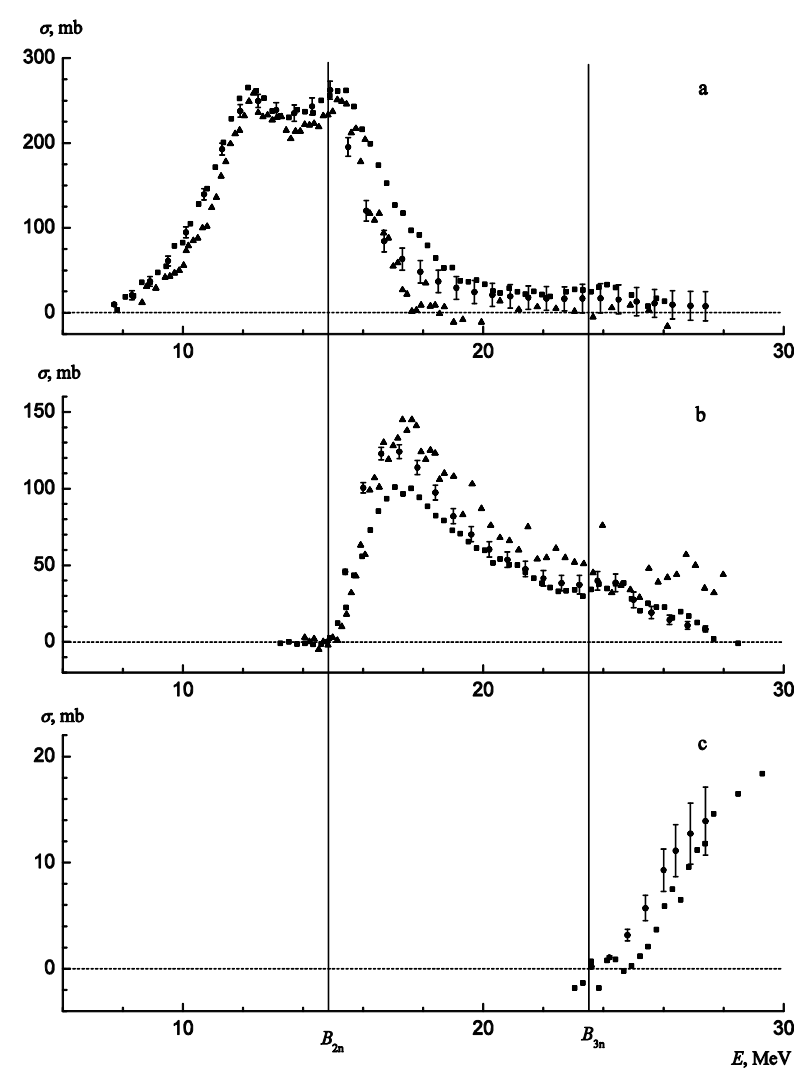

Fig. 3. Comparison between evaluated ([15], dots) and experimental (Livermore [17], triangles and Saclay [22], squares) photonuclear reaction cross sections for ${ }^{159} \mathrm{~Tb}$ : $\mathrm{a}-\sigma(\gamma, n), \mathrm{b}-\sigma(\gamma, 2 \mathrm{n}), \mathrm{c}-\sigma(\gamma, 3 \mathrm{n})$.

Table 3. Deviation of the experimental Saclay [22] and the Livermore [17] integrated cross sections data for ${ }^{159} \mathrm{~Tb}$ from evaluated ones [15].

\begin{tabular}{|c|c|c|}
\hline Reaction & $\begin{array}{c}\sigma_{\text {int }}^{\text {ival }} / \sigma_{\mathrm{S}}^{\text {int }}, \\
\%(\mathrm{MeV} \cdot \mathrm{mb})\end{array}$ & $\begin{array}{c}\sigma^{\text {int }}{ }_{\text {eval }} / \sigma_{\mathrm{L}}^{\text {int }}, \\
\%(\mathrm{MeV} \cdot \mathrm{mb})\end{array}$ \\
\hline$(\gamma, \mathrm{n})$ & $-19(1642 / 1950)$ & $+18(1642 / 1390)$ \\
\hline$(\gamma, 2 \mathrm{n})$ & $+15(715 / 610)$ & $-22(715 / 870)$ \\
\hline$(\gamma, 3 \mathrm{n})$ & $+16(26 / 16)$ & no data \\
\hline
\end{tabular}

To understand the real reasons of deviations of experimental cross section from evaluated once the correlations between such deviations and behaviour of $F_{1,2,3}{ }^{\exp }$ were investigated in details by comparison at various energies of deviations of experimental cross sections from correspondent evaluated ones.

Figure 4 presents a very sharp example of comparison of two differences $\left[\sigma^{\exp }(\gamma, \mathrm{n})-\sigma^{\text {eval }}(\gamma, \mathrm{n})\right]$ and $\left[\sigma^{\exp }(\gamma, 2 \mathrm{n})-\right.$ $\left.\sigma^{\text {eval }}(\gamma, 2 \mathrm{n})\right]$ obtained [14] for data from Livermore [9] for ${ }^{115}$ In.

One can see that the correlation is absolute - if some number of neutrons is added to $\sigma(\gamma, \mathrm{n})$ the same number of those is subtracted from $\sigma(\gamma, 2 \mathrm{n})$ and vice versa. 


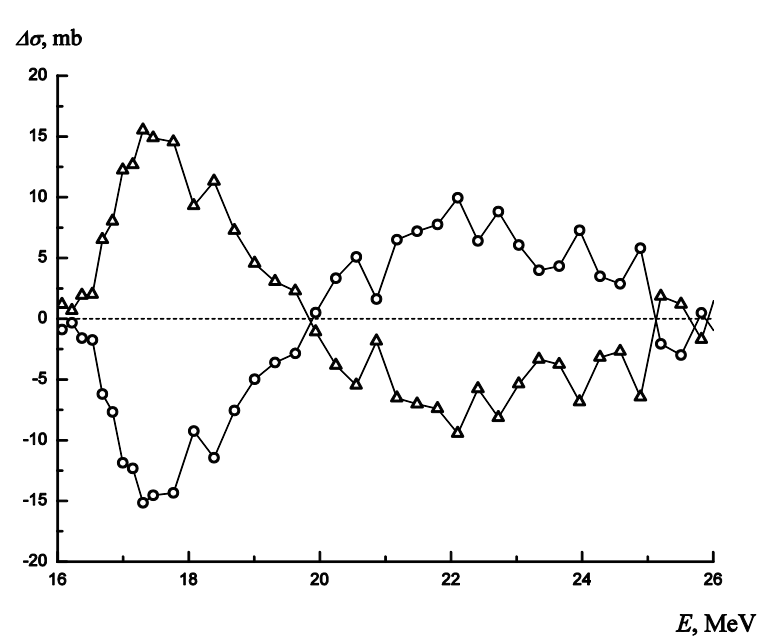

Fig. 4. Comparison of differences between experimental [9] and evaluated [14] data for ${ }^{115} \mathrm{In}$ : triangles- $\left[\sigma^{\exp }(\gamma, \mathrm{n})-\sigma^{\text {eval }}(\gamma, \mathrm{n})\right]$; circles- $-\left[\sigma^{\exp }(\gamma, 2 \mathrm{n})-\sigma^{\text {eval }}(\gamma, 2 \mathrm{n})\right]$.

We like to underline that in energy range $\sim 20-26$ $\mathrm{MeV}$ the function $F_{2}{ }^{\text {exp }}$ has (Table 2) physically unreliable values larger than 0.50 and correspondingly $\sigma(\gamma, \mathrm{n})$ has physically unreliable negative values.

It must be pointed out that mistakes in experimentally obtained neutron multiplicities depends on energy of incident photons and correspondingly of that of outgoing neutrons - at energies $\leq 20 \mathrm{MeV} \sigma^{\text {exp }}(\gamma, \mathrm{n})>\sigma^{\text {eval }}(\gamma, \mathrm{n})$. However, at energies $\geq 20 \mathrm{MeV}$ quite the contrary $\sigma^{\text {eval }}(\gamma, \mathrm{n})>\sigma^{\exp }(\gamma, \mathrm{n})$ and for $\sigma(\gamma, 2 \mathrm{n})$ the situation is quite inverse.

Absolutely the same is the situation for differences $\left[\sigma^{\exp }(\gamma, 2 \mathrm{n})-\sigma^{\text {eval }}(\gamma, 2 \mathrm{n})\right]$ and $\left[\sigma^{\exp }(\gamma, 3 \mathrm{n})-\sigma^{\text {eval }}(\gamma, 3 \mathrm{n})\right]$ in energy range $\sim 26-32 \mathrm{MeV}$ : some number of neutrons is subtracted from " $3 \mathrm{n}$ " channel (and therefore $\sigma(\gamma, 3 \mathrm{n})$ decreases and falls into the region of non-physical negative values) and corresponding number of neutrons is added to " $2 \mathrm{n}$ " channel (and therefore $F_{2}{ }^{\text {exp }}$ increases and goes to the region of non-physical values larger 0.50 ).

\section{Possible reasons of evaluated and experimental data disagreements}

\subsection{Neutron kinetic energy measurement conditions}

Both at Saclay and Livermore neutron multiplicity was obtained from neutron kinetic energy on the base of hypothesis that both neutrons from " $2 n$ "-channel have energy smaller than one neutron from " $1 \mathrm{n}$ "-channel.

However, as it was shown above the number of neutrons with small energy ("from $(\gamma, 2 \mathrm{n})$ reaction") as a rule is underestimated at Saclay but overestimated at Livermore and vice versa the number of neutrons with large energy ("from $(\gamma, n)$ reaction") is overestimated at Saclay and underestimated at Livermore. Therefore one should conclude that these disagreements could be connected with the neutron registration method.
At Saclay for neutron energy measurement the largevolume Gd-loaded liquid scintillator was used. Since the formation of two neutrons in reaction $(\gamma, 2 n)$ is realized at short nuclear time at poor time resolution of detection system there is definite opportunity for small pulses pileup that certainly could lead to increasing the number of neutrons in "1 $\mathrm{n}$ "channel in comparison to that of " $2 \mathrm{n}$ " channel.

At Livermore so-called "ring-ratio" method was used: neutron counters were putted into paraffin moderator by concentric rings around the target. Lowenergy neutrons (as have been supposed, from reaction $(\gamma, 2 n))$ should have enough time for moderation in the way from target to inner ring but high-energy neutrons (as supposed from reaction $(\gamma, n)$ ) should go to the outer ring passing inner ring. But because of multiple scattering the neutron way certainly should not be as straight line there is definite opportunity that neutron will go back to inner ring. That could certainly increase the number of neutrons in " $2 \mathrm{n}$ " channelin comparison to correspondent number of neutrons in " $1 \mathrm{n}$ " channel.

\subsection{Neutron kinetic energy and multiplicity connection}

Both at Saclay and Livermore neutron multiplicity determination methods were based on the supposition that one unique neutron from $(\gamma, n)$ reaction has energy more than both neutrons from reaction $(\gamma, 2 n)$. But as that was shown above degree of discrepancies between the Saclay and Livermore data and between both of them and new evaluated cross sections in " $1 n$, " $2 n$ ", and " $3 n$ " channels depends on energy of photons and therefore on energy of neutrons. So degree of disagreements in neutron multiplicities obtained depends on individual features of neutron spectra.

It means that neutron "energy - multiplicity" connection could be really not so simple and direct as has been supposed. In special investigation [23] it was shown that mean energy of the 1st neutron from the reaction ${ }^{181} \mathrm{Ta}(\gamma, 2 \mathrm{n}){ }^{179} \mathrm{Ta}$ is much larger than that of 2 nd neutron (for example, at photon energy $25 \mathrm{MeV} 1$ st neutron has energy 4.0 MeV, but 2nd one $-1.4 \mathrm{MeV}$ ). So both the Saclay and Livermore methods of neutron kinetic energy measurement could be mistaken generally: if the energy of first chance neutron from reaction $(\gamma, 2 \mathrm{n})$ is "enough small" the correspondent event could be correctly and reliably attributed to " $2 \mathrm{n}$ " channel, but if its energy is "enough large" the event could be erroneously and unreliably attributed to " $1 \mathrm{n}$ " channel.

Additionally that must be pointed out that really situation could be more complicated because in reaction $(\gamma, \mathrm{n})$ after escape of one unique neutron and in reactions $(\gamma, 2 \mathrm{n})$ and $(\gamma, 3 \mathrm{n})$ after escape of first chance neutron the same nucleus is formed. Moreover the same nucleus is formed in reactions $(\gamma, n p),(\gamma, n 2 p), \ldots$

Therefore the possible reason of disagreements between unreliable experimental and reliable evaluated reaction cross sections could be not direct and very complex connection between neutron kinetic energy and its multiplicity. 


\section{New evaluated data reliability and authenticity}

In section "Short history..." it was mentioned that there is alternative to neutron multiplicity sorting method of partial reaction investigation. That is the method of induced activity in which the concrete reaction is identified using detection of not outgoing neutrons but final nucleus. Because the final nuclei in reactions $(\gamma, n)$, $(\gamma, 2 \mathrm{n})$, and $(\gamma, 3 \mathrm{n})$ are different, the method of induced activity is direct for partial reaction cross section determination and there are no problems of their separation.

With the aim to test reliability and authenticity of photoneutron partial reaction cross sections evaluated in the frame of proposed new experimentally-theoretical approach special measurements were carried out [23] with the $\gamma$-quanta beam of a new generation electron accelerator - race-track microtron with the maximal electron energy $65 \mathrm{MeV}$. Using high-quality HpGe detector the yields of $(\gamma, n),(\gamma, 2 n),(\gamma, 3 n),(\gamma, 4 n),(\gamma, 5 n)$, $(\gamma, 6 \mathrm{n})$, and $(\gamma, 7 \mathrm{n})$ reactions on ${ }^{181}$ Ta were measured [23] at one experiment. Correspondent such kind data for $(\gamma, n),(\gamma, 2 n)$, and $(\gamma, 3 n)$ reactions are compared in the Table 4 with results of other experiments under discussion and data evaluated in the frame of new experimentally theoretical method proposed.

Table 4.Comparison of ratios of cross sections $\sigma$ and yields $Y$ for ${ }^{181} \mathrm{Ta}$ and reactions $(\gamma, 2 \mathrm{n}) /(\gamma, \mathrm{n})$ and $(\gamma, 3 \mathrm{n}) /(\gamma, \mathrm{n})$ obtained for experimental and evaluated data.

\begin{tabular}{|c|c|c|c|c|}
\hline \multirow{2}{*}{ Ratio } & \multicolumn{3}{|c|}{ Experiments } & Evaluation \\
\cline { 2 - 5 } & $\begin{array}{c}\text { Saclay } \\
{[22]}\end{array}$ & $\begin{array}{c}\text { Livermore } \\
{[18]}\end{array}$ & $\begin{array}{c}\text { Activity } \\
{[23]}\end{array}$ & $\begin{array}{c}\text { Our data } \\
{[24]}\end{array}$ \\
\hline $\begin{array}{c}\sigma(\gamma, 2 \mathrm{n}) / \\
\sigma(\gamma, \mathrm{n})\end{array}$ & $\begin{array}{c}0.36 \\
797 / 2190\end{array}$ & $\begin{array}{c}0.67 \\
887 / 1316\end{array}$ & & 0.49 \\
\hline $\begin{array}{c}Y(\gamma, 2 \mathrm{n}) / \\
Y(\gamma, \mathrm{n})\end{array}$ & 0.24 & 0.42 & $0.34(7)$ & 0.33 \\
\hline $\begin{array}{c}Y(\gamma, 3 \mathrm{n}) / \\
Y(\gamma, \mathrm{n})\end{array}$ & 0.02 & & $0.023(2)$ & 0.018 \\
\hline
\end{tabular}

Data presented show that in comparison with the induced activity results neutron multiplicity sorting experimental data for $\sigma(\gamma, 2 \mathrm{n})$ obtained at Saclay are definitely underestimated $(0.24$ versus 0.34$)$, those obtained at Livermore - definitely underestimated $(0.42$ versus 0.34). It is important to underline that our new data evaluated in the frame of proposed experimentallytheoretical approach agree with induced activity data well ( 0.33 versus 0.34$)$. This confirms that data evaluated in the frame of proposed new experimentally-theoretical approach are enough reliable and authentic.

However, this produces one more serious problem which should be analyzed specially. The point is that as it follows from Table 4 the data for $\sigma(\gamma, 2 n) / \sigma(\gamma, n)$ ratio obtained at Livermore for ${ }^{181} \mathrm{Ta}$ [18] are overestimated, although in special experiment has been carried many years before [7] and has used both neutron multiplicity sorting and induced activity also that was shown that $\sigma(\gamma, 2 \mathrm{n})$ obtained at Livermore is quiet reliable. Therefore it was supposed (supposed only because experimental data for $\sigma(\gamma, \mathrm{n})$ using induced activity data have not been obtained) that $\sigma(\gamma, \mathrm{n})$ for ${ }^{181} \mathrm{Ta}$ also is reliable. From the first point of view there is clear contradiction with results of our investigations using objective criteria of data reliability. To solve that contradiction the detailed evaluation has been carried out for ${ }^{181} \mathrm{Ta}$ [23] using our new experimentally-theoretical approach. The results (Table 5) were very interesting and unexpected.

Table 5.Comparison of total and partial reactions integrated cross section ratios for experimental [23] and evaluated [24] data for ${ }^{181} \mathrm{Ta}$.

\begin{tabular}{|c|c|c|}
\hline Reaction & $\begin{array}{c}\sigma_{\text {int }}^{\text {eval }} / \sigma_{\text {int }} \text { s, } \\
\text { arb.units }(\mathrm{MeV} \cdot \mathrm{mb})\end{array}$ & $\begin{array}{c}\sigma_{\text {int }}^{\text {inval }} / \sigma_{\mathrm{L}}^{\text {int }}, \\
\text { arb.units }(\mathrm{MeV} \cdot \mathrm{mb})\end{array}$ \\
\hline$(\gamma, \mathrm{xn})$ & 1 & $1.24(3814 / 3068)$ \\
\hline$(\gamma, \mathrm{sn})$ & $0.96(2867 / 2998)$ & $1.30(2867 / 2199)$ \\
\hline$(\gamma, \mathrm{n})$ & $0.88(1922 / 2190)$ & $1.46(1922 / 1316)$ \\
\hline$(\gamma, 2 \mathrm{n})$ & $1.16(929 / 798)$ & $1.05(929 / 887)$ \\
\hline
\end{tabular}

Data presented show clear that as larger is the partial reaction ${ }^{181} \mathrm{Ta}(\gamma, \mathrm{n})^{180} \mathrm{Ta}$ contribution into the total reactions $\left({ }^{181} \mathrm{Ta}(\gamma, \mathrm{xn})\right.$ and ${ }^{181} \mathrm{Ta}(\gamma, \mathrm{sn}),(\gamma, \mathrm{sn})=[(\gamma, \mathrm{n})+$ $(\gamma, 2 n)+(\gamma, 3 n)+\ldots]$ larger is deviation of that total reaction cross section from correspondent evaluated one $(1.24 \rightarrow 1.30)$. For pure ${ }^{181} \mathrm{Ta}(\gamma, \mathrm{n}){ }^{180} \mathrm{Ta}$ reaction cross section (contribution of $\sigma(\gamma, 2 \mathrm{n})$ is equal to 0 ) its deviation from correspondent evaluated one is extremely larger 1.46 ! That means that overestimation (Table 4) of ratios $\sigma(\gamma, 2 \mathrm{n}) / \sigma(\gamma, \mathrm{n})$ and $Y(\gamma, 2 \mathrm{n}) / Y(\gamma, \mathrm{n})$ for ${ }^{181} \mathrm{Ta}$ is not because $\sigma(\gamma, 2 \mathrm{n})$ namely is overestimated (moreover, data of Table 5 show that in this case we have slightly $(5 \%)$ underestimation!) but because $\sigma(\gamma, \mathrm{n})$ is extremely $(46 \%$ !) underestimated.

The reason for so significant underestimation of $\sigma(\gamma, \mathrm{n})$ is absolutely misunderstood but for ${ }^{181} \mathrm{Ta}$ this is in case really. That is very important to underline that $\sigma(\gamma, n)$ underestimation is directly reflected also in significant underestimation (Table $5-24 \%$ ) of the total neutron yield reaction cross section $\sigma(\gamma, \mathrm{xn})$ for this nucleus in comparison with not only evaluated and experimental Saclay ones but with other results of analogous cross sections obtained using bremsstrahlung [24]. It is useful to point out that in accordance with results of systematic investigations $[3,25,26]$ of discrepancies of $\sigma(\gamma, \mathrm{xn})$ reaction integrated cross section ratios obtained in various laboratories, the mean ratio for such discrepancies was estimated to be equal to $12 \%$ only.

\section{Conclusions}

Using new objective criteria of data reliability transitional multiplicity functions $F_{\mathrm{i}}$-and new experimentally-theoretical approach to photoneutron partial reaction cross section evaluation many experimental data for $(\gamma, n),(\gamma, 2 n)$ reactions (and if it was 
possible - for $(\gamma, 3 n)$ reaction) have been analyzed for 15 nuclei ${ }^{90} \mathrm{Zr},{ }^{115} \mathrm{In},{ }^{112,114,116,117,118,119,120,122,124} \mathrm{Sn},{ }^{159} \mathrm{~Tb}$, ${ }^{181} \mathrm{Ta},{ }^{197} \mathrm{Au}$, and ${ }^{208} \mathrm{~Pb}$.

It has been found out that:

- At Saclay for all nuclei mentioned above with exception of ${ }^{208} \mathrm{~Pb}$ in accordance with $F_{\mathrm{i}}$ behavior $\sigma(\gamma, \mathrm{n})$ are over- but $\sigma(\gamma, 2 \mathrm{n})$ - underestimated; for ${ }^{208} \mathrm{~Pb}$ competition of both reaction cross sections is reliable; - At Livermore for all nuclei mentioned with exception ${ }^{181} \mathrm{Ta}$ in accordance with $F_{\mathrm{i}}$ behavior $\sigma(\gamma, 2 \mathrm{n})$ are underbut $\sigma(\gamma, \mathrm{n})$ - overestimated; for ${ }^{181} \mathrm{Ta} \sigma(\gamma, 2 \mathrm{n})$ small $(5 \%)$ underestimation is correlated with significant (46\%) $\sigma(\gamma, \mathrm{n})$ underestimation;

- For ${ }^{181}$ Ta data evaluated using new experimentallytheoretical approach proposed disagree with unreliable data obtained using neutron multiplicity sorting between " $1 n$ " and " $2 n$ " channels, but agree with data obtained using alternative method of induced activity.

So the main conclusions of investigation carried out using objective criteria are the following:

- Practically all investigations show that neutron multiplicity sorting experiments were carried out incorrectly and data obtained are not reliable;

- The main reason of that is complex and not direct connection between neutron kinetic energy measured and its multiplicity obtained;

- Systematic disagreements of data obtained using various neutron multiplicity sorting methods appear from the neutron kinetic energy measurement shortcomings;

- Experimental data on partial photoneutron reaction cross sections should be reanalyzed and reevaluated individually for nuclei investigated before;

- For reliable determination of partial photoneutron reaction cross sections the methods of direct reaction identification - detection of final nucleus activity or outgoing neutrons in coincidences - should be used;

- Proposed experimentally-theoretical approach for partial photoneutron reaction cross section evaluation based on joint using of experimental data only for neutron total yield reaction cross section $\sigma(\gamma, \mathrm{xn})$ and equations of modern model of photonuclear reactions give results that contradict to the results obtained using neutron multiplicity sorting but agree to those obtained using the method of induced activity.

\section{Acknowledgements}

Authors acknowledge a great help of Drs. V.A. Chetvertkova, M.A. Makarov, N.N. Peskov, T.S. Polevich, and S.Yu. Troshchiev in data processing, and are especially thankful to Dr. M.E. Stepanov for help in data analysis and results presentation.

The work was partially supported by Russia BFR Grant 09-02-00368, Scientific Schools Grant 02.120.21.485-SS and Contract 02.740.11.0242.

\section{References}

1. B.L. Berman, S.S. Fultz, Rev. Mod. Phys. 47, 713 (1975)

2. S.S. Dietrich, B.L. Berman, At. Data \& Nucl. Data Tables 38, 199 (1988)

3. A.V. Varlamov, V.V. Varlamov, D.S. Rudenko, M.E. Stepanov, INDC(NDS)-394, IAEA NDS, Vienna, Austria, 1999.

4. Nuclear Reaction Database (EXFOR),

URL: http://cdfe.sinp.msu.ru/exfor/index.php

CSISRS and EXFOR Nuclear reaction experimental data (database),

URL: http://www.nndc.bnl.gov/exfor/exfor00.htm

5. V.V. Varlamov, B.S. Ishkhanov, N.N. Peskov, M.E. Stepanov, I.A. Pshenichnov, Phys. At. Nucl. 67, 2122 (2004)

6. U.I. Uggerhoj, I.A. Pshenichnov, et al., Phys. Rev. C 72, 057901 (2005)

7. E. Wolynec, M.N. Martins, Rev. Bras. Fis. 17, 56 (1987)

8. V.V. Varlamov, N.N. Peskov, D.S. Rudenko,

M.E. Stepanov, INDC(CCP)-440, IAEA NDC, Vienna, Austria, 2004.

9. S.C. Fultz, B.L. Berman, J.T. Caldwell, et al., Phys. Rev. 186, 1255 (1969)

10. V.V. Varlamov, B.S. Ishkhanov, V.N. Orlin,

V.A. Chetvertkova, Bull. Rus. Acad. Sci., Physics 74, 883 (2010)

11. B.S. Ishkhanov, V.N. Orlin, Phys. Part. Nucl. 38, 232 (2007)

12. B.S. Ishkhanov, V.N. Orlin, Phys. At. Nucl. 71, 493 (2008)

13. V.V. Varlamov, B.S. Ishkhanov, V.N. Orlin,

S.Yu. Troshchiev, Bull. Rus. Acad. Sci., Physics 74, 842 (2010)

14. V.V. Varlamov, B.S. Ishkhanov, V.N. Orlin, A.V. Sopov, MSU SIMP Preprint-8/864, 2010.

15. V.V. Varlamov, B.S. Ishkhanov, V.N. Orlin, Phys.

At. Nucl. 75, 2012 (to be published)

16. S.C. Fultz, B.L. Berman, J.T. Caldwell, et al., Phys.

Rev. 186, 1255 (1969)

17. R.L. Bramblett, J.T. Caldwell, et al., Phys. Rev. 133, B869 (1964)

18. R.L. Bramblett, J.T. Caldwell, G.F. Auchampaugh, et al., Phys. Rev. 129, 2723 (1963)

19. B.L. Berman, D.D. Faul, R.A. Alvarez, et al., Phys. Rev C 19, 1205 (1979)

20. R.R. Harvey, J.T. Caldwell, R.L. Bramblett, et al., Phys. Rev. 136, B126 (1964)

21. A. Veyssiere, H. Beil, R. Bergere, et al., Nucl. Phys. A 159, 561 (1970)

22. R. Bergere, H. Beil, A. Veyssiere, et al., Nucl. Phys. A 121, 463 (1968)

23. B.S. Ishkhanov, V.N. Orlin, S.Yu. Troshchiev, Phys. At. Nucl.75, 253 (2012)

24. V.V. Varlamov, V.N. Orlin, N.N. Peskov, T.S.

Polevich, MSU SIMP Preprint-1/879, 2012.

25. V.V. Varlamov, B.S. Ishkhanov, INDC(NDS)-432, IAEA NDS, Vienna, Austria, 2002.

26. V.V. Varlamov, B.S. Ishkhanov, Phys. Part. Nucl.

35, 459 (2004) 